\title{
Analysis of dense packing of highly deformed grains
}

\author{
Thi Lo Vu ${ }^{1, \star}$, Saeid Nezamabadi ${ }^{1, \star \star}$, Jonathan Barés ${ }^{1, \star \star \star}$, and Serge Mora ${ }^{1, \star \star \star \star}$ \\ ${ }^{1}$ Laboratoire de Mécanique et Génie Civil (LMGC), Université de Montpellier, CNRS, Montpellier, France
}

\begin{abstract}
This paper concerns modeling of soft granular materials in which the grains are highly deformable. In order to simulate these materials, an approach based on an implicit formulation of the Material Point Method in the context of the finite strain theory, allowing for large deformations of grains, coupled with the Contact Dynamics method for the treatment of unilateral frictional contacts between grains, is proposed. In this context, the Mooney-Rivlin constitutive relationship is applied with two different set of elastic parameters. Considering these two material behaviors, a uniaxial compression of 2D soft granular packings is analyzed. The stress-strain relation and the evolution of the packing fraction as well as of the connectivity of the grains are discussed.
\end{abstract}

\section{Introduction}

Granular materials are an important class of matters found in the nature and in a large number of industrial fields like civil engineering, food and pharmaceutical industries, powder technologies (chemistry, cosmetics...), etc. In many situations, these materials can be highly stressed and their constitutive grains undergo relatively high elastic or inelastic deformations without rupture. It means that the behavior of soft granular systems is governed by both interactions between grains (contact, adhesion...) and their individual behavior (elasticity, plasticity...) [1, 2]. However, most of experiments and numerical simulations stay in the small deformation regime for theoretical convenience and are not able to catch the behavior of granular materials with highly deformable grains [3-6].

The discrete element methods (DEM) are known as powerful and robust numerical approches in order to model the granular materials [7-9]. However, these techniques are limited in the case of hard or weakly deformable grains and take into account essentially inter-grain interactions. To allow for high deformation of grains, the continuum approaches can be applied to model grain deformability. One of these numerical models is known as Material Point Method (MPM) [10]. It is based on the discretization of each grain by a collection of material points. The information carried by the material points is projected onto a background mesh, where equations of motion are solved. The mesh solution is then used to update the material points. In order to deal with contact interactions, in previous works $[11,12]$, it is proposed to compute the normal forces and, eventually, tangential ones by taking into account the unilateral stresses and the Coulomb fric-

\footnotetext{
^e-mail: thi-lo.vu@umontpellier.fr

$\star \star$ e-mail: saeid.nezamabadi@umontpellier.fr

$\star \star \star$ e-mail: jb@jonathan-bares.eu

$\star \star \star \star$ e-mail: serge.mora@umontpellier.fr
}

tional law by means of a DEM technique, Contact Dynamics (CD) method [8].

In this paper, numerical simulation of compression of a deformable granular system is carried out by mean of Material Point Method (MPM) extended to the finite strain theory. This numerical procedure allows the soft granular materials to reach the packing fraction beyond random close packing (RCP) state. We analyze the evolution of the packing and the effect of grain shape change. As we shall see, the material behavior of the grains affects the stress level and its evolution during compaction.

\section{Numerical methodology}

In a previous work [11], an implicit formulation of the material point method has been described to model the soft granular system. It was coupled with the contact dynamics method to deal with the frictional contact between deformable grains. Based on this approach which was developed in the context of the infinitesimal strain hypothesis, we will describe briefly below its extension to the finite strain theory.

Let us consider a continuum body occupying a domain $\Omega_{0}$ in $\mathbb{R}^{D}$ ( $D$ being the domain dimension) in its reference configuration. If $\Omega$ is the actual occupied domain of this body, its conservations of mass and of linear momentum can be described by the following relations:

$$
\begin{gathered}
\frac{\partial \rho(\mathbf{x}, t)}{\partial t}+\boldsymbol{\nabla} \cdot(\rho(\mathbf{x}, t) \mathbf{v}(\mathbf{x}, t))=0 \quad \text { in } \Omega, \\
\boldsymbol{\nabla} \cdot \boldsymbol{\sigma}(\mathbf{x}, t)+\mathbf{b}(\mathbf{x}, t)=\rho(\mathbf{x}, t) \mathbf{a}(\mathbf{x}, t) \quad \text { in } \Omega,
\end{gathered}
$$

where $\rho(\mathbf{x}, t)$ is the material density, $\sigma(\mathbf{x}, t)$ denotes the Cauchy stress tensor, $\mathbf{b}(\mathbf{x}, t)$ represents the body force and, $\mathbf{v}(\mathbf{x}, t)$ and $\mathbf{a}(\mathbf{x}, t)$ are the velocity and the acceleration, respectively, at position $\mathbf{x}$ in the actual configuration and 
time $t$. The constitutive law in the framework of the finite strain theory is given by:

$$
\mathbf{P}(\mathbf{X}, t)=\mathcal{F}(\mathbf{F}(\mathbf{X}, t)),
$$

where $\mathbf{P}(\mathbf{X}, t)$ is the first Piola-Kirchhoff stress tensor and $\mathbf{F}(\mathbf{X}, t)$ denotes the deformation gradient tensor at position $\mathbf{X}$ in the reference configuration and time $t . \sigma$ is related to $\mathbf{P}$ through:

$$
\boldsymbol{\sigma}(\mathbf{x}, t)=\frac{1}{J} \mathbf{P}(\mathbf{X}, t) \mathbf{F}^{T}(\mathbf{X}, t)
$$

with $J=\operatorname{det}(\mathbf{F})$.

In the MPM, the continuum body is divided into $N_{p}$ material points with constant masses. This last assumption allows satisfying automatically the mass conservation relation (1). The material points represent the integration points to compute the integrals in the context of the finite element method. By supposing contact interactions between bodies, the discretized form of the momentum equation can be written as:

$$
\mathbf{M} \mathbf{a}_{\text {node }}(t)=\mathbf{f}^{\text {int }}(t)+\mathbf{f}^{\text {ext }}(t)+\mathbf{f}^{\mathrm{C}}(t),
$$

where $\mathbf{a}_{\text {node }}$ is the nodal acceleration, $\mathbf{f}^{\mathrm{C}}$ is the contact forces, $\mathbf{f}^{\text {ext }}$ represents the external applied force, and

$$
\mathbf{M}=\sum_{p=1}^{N_{p}} m_{p} \mathbf{N}_{p}, \quad \mathbf{f}^{\mathrm{int}}=-\sum_{p=1}^{N_{p}} \mathbf{G}_{p} \boldsymbol{\sigma}_{p} V_{p} .
$$

In the above equations, $\mathbf{M}$ is the lumped mass matrix and $\mathbf{f}^{\text {int }}$ gives the internal force vector resulting from the stress divergence. $m_{p}$ and $V_{p}$ are material point mass and volume, $\mathbf{N}_{p}$ denotes interpolation matrix or shape function matrix and $\mathbf{G}_{p}$ represents the gradient of the shape function $\mathbf{N}_{p}$ at $\mathbf{X}_{p}$.

The contact forces $\mathbf{f}^{\mathrm{C}}$ between grains are computed using a contact algorithm accounting for the condition of non-interpenetration of matter as well as the Coulomb friction law. This algorithm combined MPM and CD method was presented in detail in a previous paper [11]. Moreover, a similar MPM implicit approach as [11] is implemented to solve the problem (5). The nodal solutions are then projected onto the material points, allowing for updating the information carried by these points.

For the applications that we target in the present work, two-dimensional simulations in plane strain conditions were performed. We also consider two hyperelastic Mooney-Rivlin constitutive laws to describe the material behavior in the finite strain framework [13]. The first one is the special case of the Neo-Hookean solid (NH) with the following constitutive relation:

$$
\mathbf{S}=(\lambda \ln (J)-\mu) \mathbf{C}^{-1}+\mu \mathbf{I} .
$$

The second one, simply called below the Mooney-Rivlin (MR) model is:

$$
\mathbf{S}=2\left(c_{1}+c_{2} \operatorname{Tr}(\mathbf{C})\right) \mathbf{I}-2 c_{2} \mathbf{C}+[2 c J(J-1)-d] \mathbf{C}^{-1},
$$

where $\mathbf{S}$ is the second Piola-Kirchhoff stress tensor related to $\mathbf{P}$ through $\mathbf{P}=\mathbf{F S}$. $\mathbf{C}$ is the right symmetric Cauchy-Green tensor $\left(\mathbf{C}=\mathbf{F}^{T} \mathbf{F}\right)$, and $\boldsymbol{\gamma}$ denotes the Green-Lagrange strain tensor $\left(\boldsymbol{\gamma}=\frac{1}{2}(\mathbf{C}-\mathbf{I})\right) . \lambda, \mu, c_{1}, c_{2}$, $c$ and $d\left(d=2\left(c_{1}+2 c_{2}\right)\right)$ are the material constants.

These choices are motivated by wide range of materials that may be described by the considered constitutive laws. For instance, gels and hydrogels with deformable particles, which are present in foodstuffs, dictate to a large extent their rheological behavior [14]. Another exemple is vesicles being closed membranes which are highly deformable particles due to their internal viscous fluid and the flexibility and the quasi-inextensibility of their lipid viscous membrane [15]. On the other hand, for all applications, it is not clear to know if $\mathrm{NH}$ model is sufficient for describing the mechanical behavior of particules. Hence, in this paper, we present a comparison of obtained results between theses two constitutive laws in order to see the sensibility of granular behavior according to the choice of these laws. Note that if $c_{2}$ is set to zero and $c_{1}=\frac{1}{2} \mu$ in equation (8) without considering volumetric part of stress, we can recover the Neo-Hookean law (7). So, one can consider that MR model is an extension of $\mathrm{NH}$ one.

\section{Compaction of a deformable granular system}

Let us now study the uni-axial compression of a packing of 300 deformable circular grains modeling by means of the proposed MPM-CD procedure. This packing is confined inside a rectangular box of width $L$ and of height $h_{0}$. In the initial configuration, the grains don't touch each other and a small size polydispersity (diameters $\in[1.4,2.4] \mathrm{mm}$ ) is introduced in order to avoid long-range ordering. To avoid stress gradients, the gravity effect is neglected. There is also no friction between the grains and, the grains and the walls. Two cases are investigated below by considering two above constitutive models for the deformable grains: (i) the Neo-Hookean model expressed by Eq. (7) with $\lambda=61 \mathrm{MPa}$ and $\mu=1.26 \mathrm{MPa}$ and, (ii) the MooneyRivlin model described by Eq. (8) with the parameters $c_{1}=0.63 \mathrm{MPa}, c_{2}=-0.0012 \mathrm{MPa}$ and $c=6 \mathrm{MPa}$. Note that we chose the high value of $\lambda$ and of $c$ in order to have quasi-incompressible grains during the simulations. The compaction process is performed by moving the top wall of the box downwards at a constant velocity of $1 \mathrm{~m} / \mathrm{s}$ and a time step of $\delta t=0.1 \mu \mathrm{s}$.

We are particularly interested here in the grain shape change due to the individual grains behavior, which may allow the packing fraction $\Phi$ to exceed the RCP state. Indeed, $\Phi$ being the ratio of the particles volume $V_{s}$ to the total volume $V: \Phi=V_{s} / V$, varies due to both the volumetric particle deformation $\Delta V_{s} / V_{s}$ as a result of the compressibility of grains and the variation of $V$ due to grain rearrangements and shape change:

$$
\ln \left(\frac{V_{s}}{V_{S_{0}}}\right)=\ln \left(\frac{\Phi}{\Phi_{0}}\right)+\varepsilon
$$




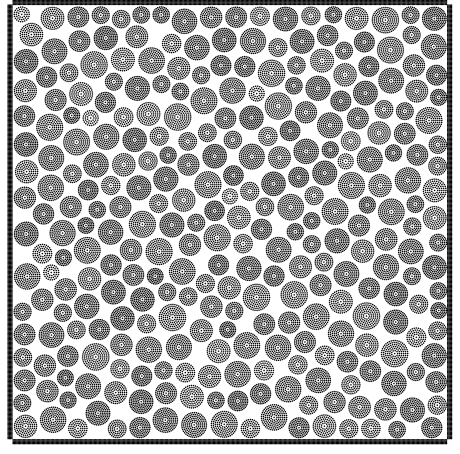

(a) $\varepsilon=0$ and $\Phi=0.65$

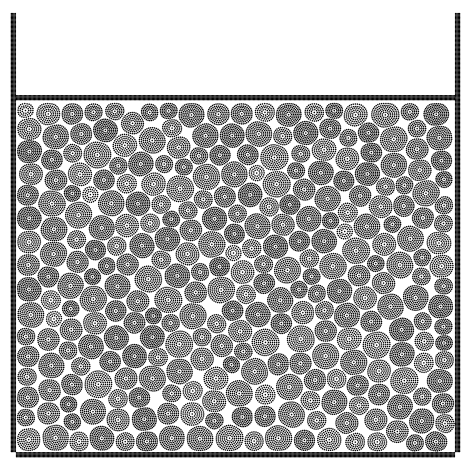

(b) $\varepsilon=0.22$ and $\Phi=0.8$

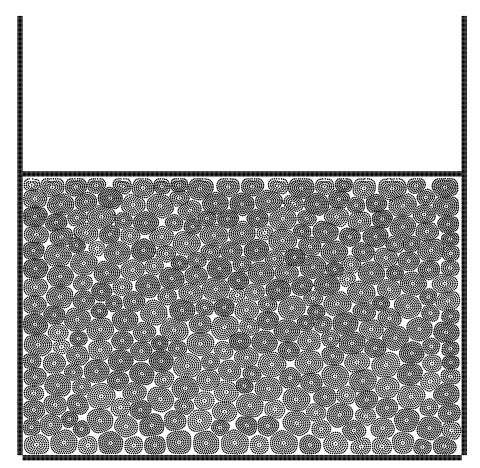

(c) $\varepsilon=0.45$ and $\Phi=0.99$

Figure 1: Three snapshots of the compaction of a packing of deformable grains. Filled Circles are material points.

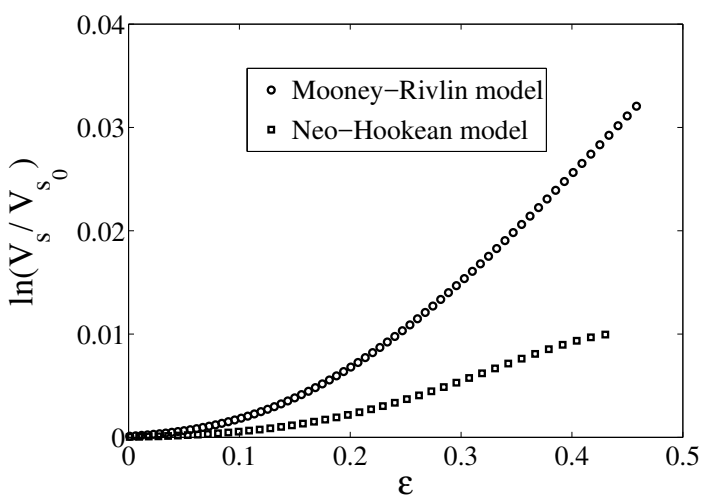

Figure 2: Total particles volume change $\ln \left(V_{s} / V_{s_{0}}\right)$ as a function of the cumulative vertical strain $\varepsilon$.

where $\varepsilon=\ln \left(V / V_{0}\right) \equiv \ln \left(h / h_{0}\right)$ is the true cumulative vertical strain. The evolution of $\ln \left(V_{s} / V_{s_{0}}\right)$ with $\varepsilon$ is represented in Fig. 2. Since, at the beginning, the compaction is mainly governed by the grain rearrangements, $\ln \left(V_{s} / V_{s_{0}}\right)$ is quasi-null. The cumulative volume deformation varies approximately linearly with $\varepsilon$ when $\varepsilon \in[0.1,0.22]$. In this range, the grain strains are due to contact deflections, as in the Hertz regime. The 'jamming' transition occurs for $\varepsilon \simeq 0.22$ and beyond this value of the strain, the individual grain deformations are mainly dictated by the packing deformation. So, the volumetric deformation of the grains increases at a higher rate. We also observe that the MR grains semble more compressible than the $\mathrm{NH}$ ones. It may be explained by the fact that the ratio of $\lambda / \mu$ is larger than $c / c_{1}$. Indeed, these ratios are controlled the compressibility of the grains. However, the cumulative volume change of the grains remains small for both cases $\left(\ln \left(V_{s} / V_{s_{0}}\right)<4 \%\right)$ and one can claim that the MR and $\mathrm{NH}$ grains are quasi-incompressible.

Fig. 3 shows the packing fraction $\Phi$ as a function of cumulative strain $\varepsilon$. $\Phi$ is a nearly linear function of $\varepsilon$ for both cases since the grains volume approximately does not vary: $\Phi=\Phi_{0}^{\prime} \mathrm{e}^{\varepsilon} \simeq \Phi_{0}^{\prime}(1+\varepsilon)\left(\Phi_{0}^{\prime}=0.65\right)$. Nevertheless, we

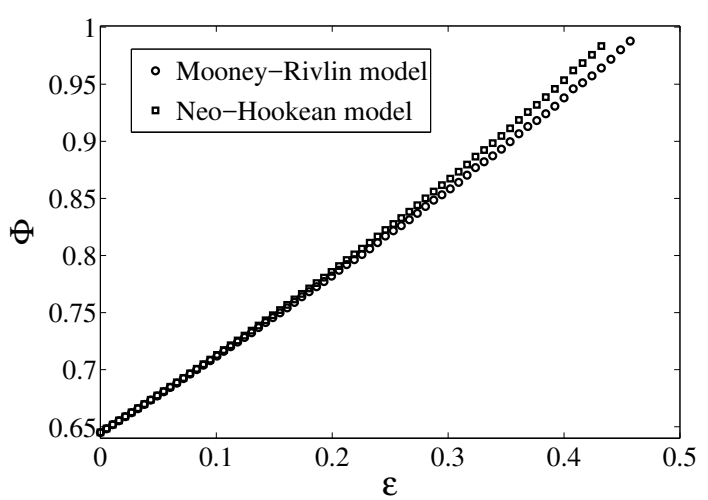

Figure 3: Packing fraction $\Phi$ as a function of cumulative vertical strain $\varepsilon$.

observe a little difference between the two results for $\varepsilon>$ 0.22 , i.e. the regime of the individual grains deformation. It is due to the difference in the compressibility of the MR and $\mathrm{NH}$ grains.

The macroscopic evolution of the packing can be tracked through the vertical stress $\sigma$ from the jamming point at $\varepsilon \simeq 0.22$ where $\Phi \simeq 0.8$. Fig. 4 represents $\sigma$, computed from the forces acting on the bottom wall and normalized by a consolidation stress $\sigma_{0}$, as a function of the cumulative vertical strain $\varepsilon^{\prime}$ from the reference state defined by $\sigma=\sigma_{0}$. The consolidation stress $\sigma_{0}$ corresponds to the jamming point. Similar behaviors are observed for both MR and NH grains. Nevertheless, we note a divergence of the results for $\varepsilon^{\prime}>0.05$. It can be explained by the difference behavior of the grains in two cases.

In order to analysis the microscopic evolution of the soft granular packings, we consider the variation of the mean coordination number $Z$ as a function of the packing fraction $\Phi$; see Fig. 5. We observe that $Z$ increases with $\Phi$. It is remarkable to note that the results for two cases are superimposed by normalizing $Z-Z_{0}$ per $Z_{1}-Z_{0}$ and $\Phi-\Phi_{0}$ per $\Phi_{1}-\Phi_{0}$, where $Z_{0}$ is the coordination number corresponding to $\Phi_{0} \simeq 0.8$ (jamming transition) and $Z_{1}$ 


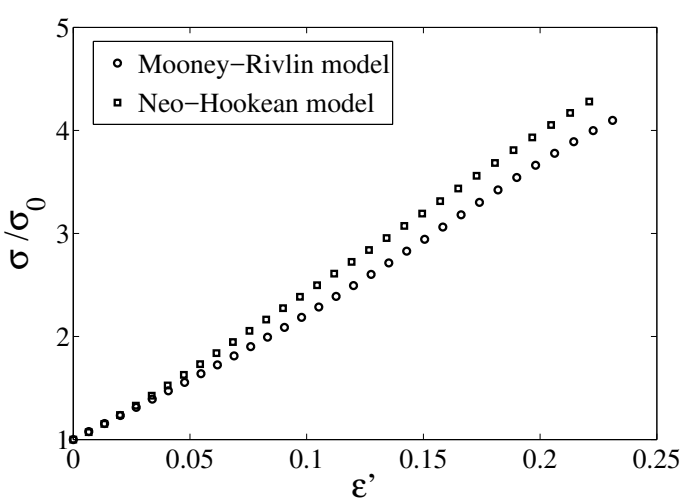

Figure 4: Normalized axial stress $\sigma / \sigma_{0}$ as a function of cumulative strain $\varepsilon^{\prime}$ from the reference state defined by $\sigma=\sigma_{0}$.

for $\Phi_{1} \simeq 1$. One can proposed the following power law relation to fit these data points as shown in Fig. 5:

$$
\frac{Z-Z_{0}}{Z_{1}-Z_{0}}=\left(\frac{\Phi-\Phi_{0}}{\Phi_{1}-\Phi_{0}}\right)^{0.5}
$$

This power-law dependance of the coordination number with the packing fraction is almost independent of dimension, interaction potential or polydispersity $[2,6,16]$. It is worth noting that in the above relation, there is no adjusting parameter contrary to the previous works (see for example [16]) and it fits quite well the results of the MR and $\mathrm{NH}$ grains.

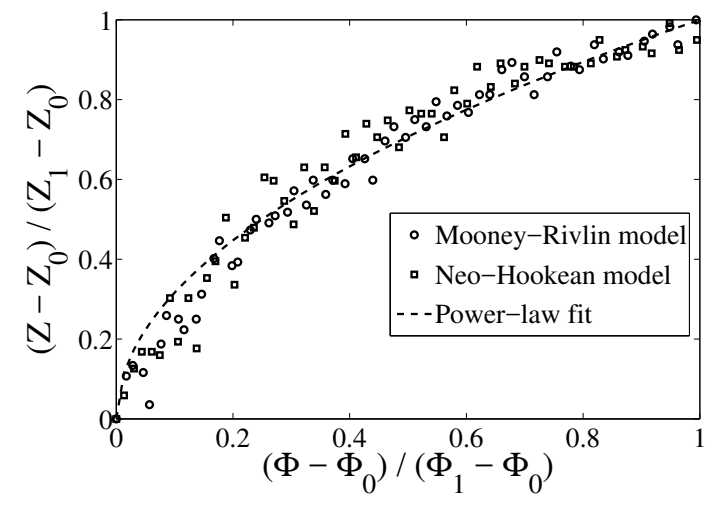

Figure 5: Evolution of the mean coordination number $Z$ as a function of packing fraction $\Phi$. The line is a power law fit $\left(\Phi-\Phi_{0}\right)^{0.5}$.

\section{Conclusion}

In this paper, the uniaxial compression of a deformable granular packing is studied using an extended approach of the MPM-CD procedure [11] in the context of the finite strain theory. This procedure combined the material point method (MPM) with the contact dynamics (CD) method. Two study cases are investigated by considering two hyperelastic constitutive relations: Neo-Hookean $(\mathrm{NH})$ and Mooney-Rivilin (MR) models. Because of the material parameters choices, the MR grains are more compressible than $\mathrm{NH}$ ones although one can assume that both types of grains are quasi-incompressible. However, this little difference in the compressibility affects the behavior of the assemblies of soft grains. So, it may be interesting to study more the compressibility effects in a future work. Another important point concerns a simple power law which relates the coordination number directly to the packing fraction without any correction pre-factor. This point also merits further analysis by considering other types of material behavior.

\section{Acknowledgment}

This work has been carried out thanks to the support of the LabEx NUMEV project (no ANR-10-LABX-20).

\section{References}

[1] K. Liu, Journal of Physics D: Applied Physics 39, 189 (2006)

[2] M. van Hecke, Journal of Physics: Condensed Matter 22, 033101 (2010)

[3] F. Radjai, M. Jean, J.J. Moreau, S. Roux, Physical Review Letters 77, 274 (1996)

[4] F. da Cruz, S. Emam, M. Prochnow, J.N. Roux, F. Chevoir, Physical Review E 72 (2005)

[5] T.S. Majmudar, R.P. Behringer, Nature 435, 1079 (2005)

[6] J. Zhang, T.S. Majmudar, M. Sperl, R. Behringer, Soft Matter 6, 2982 (2010)

[7] P.A. Cundall, O.D.L. Strack, Géotechnique 29, 47 (1979)

[8] J.J. Moreau, European Journal of Mechanics A Solids 13, 93 (1994)

[9] F. Radjai, F. Dubois, eds., Discrete-element Modeling of Granular Materials (Iste-Wiley, London, 2011)

[10] S. Bardenhagen, J. Brackbill, D. Sulsky, Computer Methods in Applied Mechanics and Engineering 187, 529 (2000)

[11] S. Nezamabadi, F. Radjai, J. Averseng, J.Y. Delenne, Journal of the Mechanics and Physics of Solids 83, 72 (2015)

[12] S. Nezamabadi, T. Nguyen, J.Y. Delenne, F. Radjai, Granular Matter 19, 8 (2017)

[13] S. Nezamabadi, H. Zahrouni, J. Yvonnet, Computational Mechanics 47, 77 (2011)

[14] G. Lorenzo, N. Zartizky, A. Califano, Food Hydrocolloids 30, 672 (2013)

[15] M. Abkarian, A. Viallat, Soft Matter 4, 653 (2008)

[16] C. O’Hern, L. Silbert, A. Liu, S. Nagel, Physical Review E 68, 011306 (2003) 